\title{
El análisis comparativo de las Diplomacias de Defensa española y rusa ${ }^{1}$
}

\author{
Comparative analysis of the Spanish and Russian \\ Defense Diplomacy
}

\author{
Arina A. Andreeva ${ }^{2}$
}

Resumen: La idea principal del artículo se centra en el análisis del concepto de la Diplomacia de Defensa. Como punto de partida fue elegida la versión española, la que se compara con las políticas exteriores rusas en el ámbito de la defensa internacional. Se hace hincapié en que la adopción de una versión global sobre los agentes que interactúan en los problemas de la seguridad, incorporación del Ministerio de Defensa en la acción exterior del Estado, hoy en día responde eficazmente a las demandas del ambiente internacional. Rusia, carente de jure del plan de la Diplomacia de Defensa, y tomando en consideración de que en 2020 vencen todas sus estrategias esenciales de la política exterior y de defensa, está en el momento de redefinir sus métodos y enfoques. La presente investigación pretende arrojar algo de luz sobre las modalidades de la Diplomacia de Defensa existentes, y sobre el por qué es tan importante la elaboración de dicho concepto nacional.

Palabras clave: Diplomacia de Defensa, seguridad, Fuerzas Armadas, política exterior, imágen en la arena internacional, España, Rusia.

Abstract: This article focuses on the analysis of the concept of Defense Diplomacy. The Spanish version was chosen as a starting point, and is compared with the Russian foreign policies in the field of international defense. It is emphasized that the adoption of a global version about the agents that interact in security problems, incorporating the Ministry of Defense into the State's foreign action, nowadays responds effectively to the demands of the international environment. Russia fails to have a de jure Defense Diplomacy plan, and taking into account that in 2020 all its essential foreign and defense policy strategies expire, it is time to redefine its methods and approaches. This research aims to shed some light on the modalities of the existing Defense Diplomacies and on why the formulation of such a national concept is so important.

Key words: Defense Diplomacy, security, Military Forces, foreign policy, image in international arena, Spain, Russia.

\footnotetext{
DOI: $10.24215 / 23142766 \mathrm{e} 096$

${ }^{1}$ Recibido 06/02/20 - Aceptado 23/06/20

2 Profesora titular, Universidad Rusa de la Amistad de los Pueblos, Facultad de Economía, Cátedra de Estudios Iberoamericanos, Moscú, Rusia. Investigadora Senior, Instituto de Latinoamérica de la Academia de Ciencias de Rusia, Centro de Estudios Ibéricos, Moscú, Rusia. andreeva_aa@pffur.ru
} 


\section{Introducción}

Al finalizar la Guerra Fría (1946-1988) y con la disolución del pacto de Varsovia (1991) por un momento pareció que la lucha entre los dos grandes bloques, con los centros en Moscú y Washington, desapareció. Debía empezar la época marcada por la cooperación en pos de la paz universal sin rivalidades. Las grandes potencias mundiales, junto con las potencias regionales, tenían que sumarse a la causa noble para garantizar la estabilidad del sistema internacional y hacer frente a los problemas globales que se avecinaban. En esa época se cristalizó la percepción de cuál sistema socio-económico ganó "la batalla" y de cuáles ideales, a partir de entonces, hacían falta alcanzar. (Fukuyama, 1989). Para lograr esos "modelos perfectos", la política exterior de los Estados, que se articula a través de los corpus diplomáticos, tenía que adaptarse a los nuevos tiempos, a sus demandas y al nuevo orden mundial.

También es cierto que los años 90 del siglo pasado no fueron de los tranquilos. Al contrario, ninguna década había visto el despliegue de tantas operaciones de paz a escala global hasta el momento. $Y$ esas misiones tampoco eran típicas: los conflictos en Centroamérica ${ }^{3}$ y en los Balcanes ${ }^{4}$ no se desarrollaban según el escenario clásico. Es decir, no fueron conflictos originados por las tensiones interestatales. Sus causas estaban intrínsecamente ligadas a los choques socio-económicos y problemas institucionales estructurales internos. Las Fuerzas Armadas (FAS) internacionales que estaban involucradas en dichas misiones se vieron obligadas a aprender nuevas "estrategias", por así llamarlas, como son la negociación, la cooperación con instituciones y actores civiles y, además, la reconstrucción post conflicto.

Como un acontecimiento de fondo, se abrían en progresión las agregadurías militares en las misiones diplomáticas en el extranjero. A simple vista, uno puede llegar a la conclusión lógica de que los Ministerios de Defensa hace treinta años diversificaron sus campos de acción con las tareas más propias del "poder blando" (en el sentido de cooperación y construcción de los lazos de confianza). Pero la historia es algo más compleja e intrincada, y nos obliga a introducir matices. La verdad es que el primer intercambio de los consejeros de guerra sucedió entre Francia y Rusia en el siglo XIX. Y el empeño de las FAS de apaciguar los enfrentamientos tampoco es nuevo. Respecto al tema, Félix Sanz Roldán (2000: 519) escribe que "el empelo de los medios militares para evitar que se produzcan conflictos, o para darles solución en los momentos iniciales de su desarrollo, se ha aplicado durante toda la historia".

Lo destacado (y pionero) de los años 1990 resultó ser la incorporación del aspecto militar en la política exterior del Estado. Sucedió el hecho por primera vez en el Reino Unido en 1998 (The Strategic Defense Review White Paper, 1998), lo que no es casual dada la larga y sofisticada trayectoria diplomática que ha experimentado este país en el transcurso del tiempo (Cosialls Ubach, 2016:25). La Diplomacia de Defensa británica contenía una variada serie de actividades preparadas para el Ministerio de Defensa y orientada a la prevención de conflictos, solución de crisis (inclusive en el momento de su desarrollo), prevención de la

\footnotetext{
${ }^{3}$ Misiones de paz de la Organización de Naciones Unidas en América Central (1989-1991), en Haití (19901991), en Salvador (1991-1996) y en Guatemala (1995-2003).

${ }^{4}$ Operaciones de la OTAN a partir de 1992.
} 
proliferación del armamento de destrucción masiva (ADM), cooperación antiterrorista, cooperación para tratar los temas de seguridad energética, cooperación para tratar las amenazas difíciles de predecir en el futuro, fortalecimiento de las medidas de confianza entre los aliados y socios, desarrollo de la metodología universal para evaluar el entorno estratégico y maneras de reacción a sus cambios, respaldo político a las operaciones colectivas en el presente y en el futuro (Tuzhikov y Pavlov, 2016:70).

Cuatro años más tarde, un documento parecido fue desarrollado por Francia (Doctrine interarmées sur la prévention. Prévention des crises et diplomatie de défense, 2002). Según Ignacio Azuara Blanco (2009: 81), los propósitos de ambas diplomacias se centraban en "disipar la hostilidad, fomentar la confianza y asegurar el control democrático de las FAS". Salvo una excepción, que "a diferencia de las "magnánimas" intenciones de la diplomacia de defensa británica, en Francia no se ocultaba que la suya se enfoca a desarrollar una capacidad de influencia sobre el ambiente internacional" (Ibíd., p. 82).

Con base en las ideas introducidas en los apartados anteriores, se puede añadir que la Diplomacia de Defensa, al unirse a la Política exterior del Estado, se ha visto transformada en el "poder inteligente", explicado por Suzanne Nossel (2004) como "el empeño de fortalecer los intereses (nacionales) a través de la red estable de aliados, instituciones y normas". Una vez más hay que reiterar que estos empeños y aspiraciones no son nuevos; la calidad distintiva entre el presente y el pasado se halla en creación del sistema coordinado entre distintos ministerios y agentes, por lo que el resultado de su trabajo debe tener el mayor impacto.

\section{La finalidad y la estrategia metodológica del artículo.}

El objetivo central del artículo es demostrar que la ejecución práctica de la Diplomacia de Defensa, al igual que la ejecución de la diplomacia clásica, puede contribuir a la seguridad internacional, al fortalecimiento del diálogo político y a la imagen positiva del país. Ante una falta de la teoría desarrollada y asentada sobre el concepto en cuestión, y con escasas publicaciones analíticas, también se pretenderá demostrar su valor para la Teoría de Relaciones Internacionales. Como la categoría de "poder" desempeña un rol central para caracterizar las relaciones entre los Estados (diferenciando su articulación en la versión "dura", coerción, y "blanda", persuasión), la hipótesis consiste en que la Diplomacia de Defensa puede ubicarse entre estos dos extremos y simbolizar el desarrollo de otro tipo de poder: el "poder inteligente". En otras palabras, el "poder blando, ejecutado clásicamente a través del atractivo de la cultura, la imagen y la confianza que goza un país en la arena internacional, y cuya promoción siempre ha estado ligada al trabajo de los corpus diplomáticos, con el desarrollo de la Diplomacia de Defensa, pasa también a las manos de las FAS. Su profesionalidad, la lealtad a los compromisos, y los valores estatales que protegen y por los que velan pueden ayudar a entrelazar las relaciones de confianza entre Ministerios de Defensa de distintos países. Por otra parte, la naturaleza de las FAS se describe por los métodos de acción rígidas, lo que les convierte también en el titular del poder duro.

En el marco de la hipótesis, y dados los obstáculos que ha enfrentado la política exterior rusa, especialmente con la defunción de la imagen negativa, resulta interesante comparar los recursos de los que dispone y los métodos que emplea un país, que se enfrenta a las 
ofensivas de desacreditación (Rusia), con los recursos y métodos de otro Estado, que ya había hecho frente a los problemas parecidos (España). Asimismo, el artículo en su análisis solo se centrará en el recurso de la Diplomacia de Defensa llevado a cabo por España y por Rusia.

Para cumplir con los propósitos planteados, en primer lugar, se recurrirá a los documentos constituyentes (El Plan de Diplomacia de Defensa de España de 2011) y, a falta de su análogo ruso, a los documentos por los que se rige la política exterior rusa (la mayoría fue elaborada entre 2014 y 2016). El Plan español será trazado en varios apartados temáticos y se pretenderá buscar sus análogos rusos. En ambos casos, serán igualmente utilizados los datos proporcionados por los Ministerios de Defensa, los Ministerios Exterior y las ramas de ejército (terrestre, naval, aéreo y de emerjencias) y los portales sobre actas del derecho internacional. En el anexo se publicarán tablas para mostrar en qué aspectos de defensa los países cooperan y qué zonas geográficas son más prioritarios para estos países.

\section{La realización de la Diplomacia de Defensa en España.}

España estuvo entre las filas de aquellos países que, antes de redactar su Diplomacia de Defensa, habían practicado muchas formas de ella, como son: ayudas militares, consultas en asuntos de seguridad y defensa, asistencia a reformas del sector de seguridad y procesos democráticos, preparación de especialistas militares, contribución durante catástrofes humanitarias y desastres naturales, etc. Siguiendo el ejemplo de otros Estados, el Plan de Diplomacia de Defensa español fue elaborado en 2011. Su contenido se puede estructurar en cuatro grupos: reuniones de los delegados especiales de ministerios, misiones de paz, formación y ejercicios conjuntos, y tecnologías de la defensa compartidas.

En el primer grupo entran reuniones de alto nivel, actividades de agregados militares, participación en marchas militares, exposiciones, ferias, etc., trabajo de comisiones mixtas (al cabo de las cuales se firma tratados de cooperación militar) y grupos de expertos. En otras palabras, son acciones para fomentar la imagen pública y la presencia de España en el extranjero. En el "Anexo 1" se aporta la estadística de tratados en materia de seguridad firmados por España con otros países, que abarca ámbitos de muy distinta índole, como desde convenios militares hasta acuerdos de protección civil.

El segundo grupo, misiones de paz, está estrechamente relacionado con el tercero (formación y ejercicios conjuntos). Cabe destacar que dichas operaciones se llevan a cabo aparte de los compromisos de seguridad colectiva asumidos por España ante la Organización del Atlántico Norte (OTAN), la Unión Europea (EU) o la Organización de Naciones Unidas $(\mathrm{ONU})$, y suponen más iniciativa y rendimiento para España, ya que todas ellas se correlacionan con la zona prioritaria para la seguridad del país, que es África. Esta es la única "región de caos", frente a las zonas estables del norte, este y oeste, con la que España tiene fronteras físicas. Las amenazas aquí emanan de la inmigración masiva ilegal, del terrorismo yihadista y del narcotráfico (Misiones en el exterior, 2020). Para combatir estos desafíos hace falta tener de socios Estados fuertes con el sector de seguridad sólido. Pero, por desgracia, muchos de los países africanos son deficientes en dicha materia. Es por eso que junto 
a las operaciones en la zona (en el Golfo de Guinea ${ }^{5}$ o en el marco del programa de Seguridad Cooperativa con Mauritania, Senegal y Túnez ${ }^{6}$ ) se aplica el denominado "plan de reforma del sector de seguridad". Dicha reforma tiene como el objetivo la modernización de las Fuerzas Armadas para que estas puedan ejercer sus funciones con más calidad. Esto se traduce o bien en su entrenamiento o bien en transformaciones institucionales, como es la creación de nuevas estructuras específicas o el perfeccionamiento de la normativa jurídica. $Y$ es aquí cuando estas metas se combinan con las actividades del grupo tres (formación).

Continuando la línea de pensamiento del párrafo anterior, cabe añadir que si bien la formación en el marco de los programas de Seguridad Cooperativa está destinada a un país concreto y se realiza a través de los convenios específicos, firmados previamente en las comisiones mixtas, la política de formación de los militares extranjeros, como tal, está ausente de estos tratados y se realiza en el territorio español. El idioma de trabajo, por supuesto, es el castellano y, como es lógico, la mayoría de los alistados son de los países del habla hispana. Es más, el Instituto Cervantes también desempeña función de la entidad colaboradora al ofrecer los cursos de español gratis para los militares en los países donde tiene sus sucursales (Moliner González, 2016:86).

La formación de los militares extranjeros persigue un triple objetivo. El primero no es nada sofisticado: consiste en dar a conocer la experiencia española sobre cómo actuar en situaciones de crisis, el funcionamiento de las estructuras de seguridad y de la defensa. Aparte están los cursos de la cuarta rama del ejército, Unidad Militar de Emergencias (UME), sobre artefactos explosivos, catástrofes y desastres naturales. También existen programas para los altos cargos militares ${ }^{7}$ (Bartolomé). El segundo objetivo es el que va de la mano de una manera natural: es el contacto personal entre los militares, posibilidad de conocer la cultura de otros países, las diferencias valorativas (si es que existen), tejer lazos de amistad. Y el tercer objetivo empuja la colaboración en el ámbito de la industria militar al informar de los avances tecnológicos españoles en este sector.

El cuarto y último grupo, representado por la industria militar, por un lado, tal y como lo justamente apunta Ignacio Azuara (2009), con toda la lógica de la palabra "diplomacia",

\footnotetext{
${ }^{5}$ El programa de vigilancia en el Golfo de Guinea empezó en 2014 y supone el control del territorio marítimo por las fuerzas españolas para promover la seguridad. Las amenazas aquí la representan terroristas y grupos criminales organizados que se dedican a la piratería, narcotráfico y tráfico de personas. Otra actividad de las FAS está relacionada con los ejercicios conjuntos con los países de la región, tales como Angola, Mauritania, Gabón, Ghana, Senegal, Camerún, Cabo Verde o Costa de Marfil, con el objetivo de formar cuerpos nacionales que puedan controlar efectivamente sus respectivas zonas marítimas.
}

${ }^{6}$ El tratado de seguridad colectiva con Mauritania, firmado en 2015, se centró en el adiestramiento y en la ayuda técnica. El plan de ejercicios conjuntos con las FAS senegaleses (2014) contenía formación en lucha cuerpo a cuerpo, contra terroristas, así como en la búsqueda y rescate y en la reconstrucción de infraestructuras. El adiestramiento en el caso de Túnez (2016) se llevó a cabo en seis áreas: reconocimiento táctico, combate en desierto, artefactos explosivos artificiales, operaciones especiales, vigilancia marítima y servicio militar de sanidad. Después de 2017 no hay constancia de datos sobre los ejercicios conjuntos con dichos países, por lo que se puede deducir que los tratados de seguridad cooperativa han concluido.

${ }^{7}$ Desde 2007 cada año se lleva a cabo un curso para los militares afganos en temas de estrategia, normativa jurídica, industria militar española y vías del desarrollo de la política de defensa nacional. Según los datos de 2016, a esos cursos también asisten representantes de la zona del Océano Pacífico, Norte de África, Oriente Próximo e Iberoamérica. 
no puede considerarse parte del concepto del estudio del artículo presente. A simple vista, entendemos por la diplomacia las actividades de negociación, de buena fe, con el objetivo de promover la imagen del país en la arena internacional y, por consiguiente, adquirir más poder e influencia en la toma de decisiones en asuntos mundiales (o regionales). La industria militar tiende más a ubicarse en el área del comercio. No obstante, el ejercicio de las actividades anteriores tiene el impacto directo en el desarrollo de colaboración armamentística. Los acuerdos de la Seguridad Cooperativa pueden suponer la ayuda técnica. Asimismo, presentar las posibilidades y novedades tecnológicas de defensa españoles forma parte de los cursos de enseñanza militar. Es más, respecto al potencial de cooperación en materia de defensa para cobrar más importancia como actor planetario, Andrey Gubin citando a Richard Bitzinger (2016: 66), escribe lo siguiente:

Se considera tradicionalmente que las exportaciones del armamento con más frecuencia se utilizan como herramienta para lograr los objetivos estratégicos militares. Es decir, fortalecer relaciones de socios, apoyar a los aliados, incrementar el grado de interacción entre las fuerzas armadas de distintos países. Al mismo tiempo, el suministro de materiales militares pueden considerarse un instrumento eficaz para demonstrar el avalo geopolítico, incluso, garantizar la seguridad del Estado-beneficiario.

En España, habitualmente, todos los tratados de producción y tecnologías militares se firman aparte (ver el Anexo 1). El Ministerio de Defensa (la Secretaría General de Política de Defensa) en ese caso representa un eslabón entre la Dirección General de Armamento y Material, por un lado, y el "mercado internacional", por el otro, reservando para sí la función reguladora. Según los datos del Ministerio de Industria, Comercio y Turismo de España, a partir del año 2011, cuando fue adoptado el Plan de Diplomacia de Defensa, las ventas de los materiales militares y de doble uso se duplicaron ${ }^{8}$. Los principales consumidores de estos materiales fueron los países con los que España desarrolla proyectos comunes en dicho ámbito: Italia, Francia, Alemania y el Reino Unido. Los estados de la UE y la OTAN representan más del $75 \%$ del mercado, los países-consumidores más grandes que no pertenecen a estas organizaciones fueron Arabia Saudí, Turquía y Singapur (Exportaciones españolas de material de defensa, 2019).

\section{El caso ruso.}

La política exterior rusa se rige sobre la base de seis documentos principales: el Concepto del desarrollo socio-económico a largo plazo (2008-2020), la Estrategia de la seguridad nacional (2015), la Doctrina Militar (2014), el Concepto de la política exterior (2016), la Doctrina Naval (2015), la Estrategia del desarrollo de la zona Ártica (2014-2020). De jure la Diplomacia de Defensa no figura entre los tipos de la diplomacia reconocidos y aplicados por dichos

\footnotetext{
${ }^{8}$ La cifra máxima antes de 2011 alcanzó algo menos de 1,5 millones de euros, mientras que después de esa fecha, la cifra de las ventas más bajas no igualó este nivel, y en 2013 el valor de las exportaciones llegó a casi 4 millones de euros.
} 
conceptos, doctrinas y estrategias ${ }^{9}$. Esto nos lanza atrás en el sentido, cuando en un país dado sí que existen las prácticas propias al concepto en cuestión, pero las iniciativas se encuentran aisladas y carecen de la visión general incorporada en la acción exterior. Además, hasta la fecha de hoy en Rusia solo ha habido un estudio acerca del tema, una mesa redonda organizada en 2016 por la Universidad Estatal de San Petersburgo. Apoyándose en las reflexiones de Alexei Podberezkin (2016: 16), podemos decir que tal vez eso se debe a que la sociedad rusa en su mayoría (tanto las élites, como los investigadores y los ciudadanos) todavía asocia a la diplomacia con el concepto tradicional, considerada esta como herramienta política contrapuesta a la acción militar, en el sentido de que los objetivos políticos se logran gracias a la fuerza militar (o amenaza de su uso) mientras que la diplomacia simplemente da forma a los resultados del enfrentamiento (a través de tratados, convenios, etc.).

No obstante, con la aparición de otras formas de la lucha sin el uso de la fuerza clásica, como causa del desarrollo de las tecnologías de información y telecomunicación, el análisis y los intentos de considerar lo que puede, en el caso ruso, agruparse en la denominada Diplomacia de Defensa tiene su relevancia. Además, entendiendo la realidad que ahora vive el país, cuando todos sus seis conceptos mencionados arriba vencen este mismo año, es decir en 2020.

Teniendo en cuenta las dimensiones físicas, el potencial tanto de recursos naturales, como el capital humano, las capacidades y la prioridad que se le otorga en Rusia a las tecnologías militares, el posicionamiento del armamento nuclear y el lugar en el escenario geopolítico mundial, el Plan de Diplomacia de Defensa de España puede servir a Rusia como un punto de partida, pero eso no significa convergencia absoluta en los campos de acción para el Ministerio de Defensa ruso.

Tanto la Estrategia de la seguridad nacional, como la Doctrina Militar y el Concepto de la política exterior, entre los países prioritarios para la cooperación, destacan los pertenecientes a las organizaciones: BRICS (Brasil, Rusia, India, China, África del Sur), la Comunidad de los Estados Independientes (CEI), Organización del Tratado de Seguridad Colectiva (OTSC), Organización de Cooperación de Shanghai (OCS), G-20. La Unión Europea y la OTAN figuran al final de la lista y se citan en relación con "su expansión geopolítica", es decir, en forma de crítica. O bien, para hacer el hincapié que sin colaboración con ellos no se puede resolver los problemas de suma importancia mundial (Doctrina Militar de la Federación Rusa, 2014). Este enfriamiento de relaciones, a pesar de la vecindad geográfica y del peso de dichas organizaciones como actores políticos en la escena mundial, se debe a los acontecimientos originados en Ucrania y Crimea en 2014, cuando los países occidentales en el conflicto ruso-ucraniano ocuparon lados opuestos.

Como se puede apreciar en el Anexo 2, el primer acuerdo en materia de cooperación en el ámbito de defensa fue firmado en 1997 con Bielorrusia (con Tayikistán, aunque concluido en 1994, entró en vigor en 2001). Hasta 2013 fueron negociados solo once tratados, pero a partir de aquel año se activó progresivamente la firma de nuevos convenios que,

\footnotetext{
${ }^{9}$ Los tipos de la diplomacia reconocidos son: diplomacia pública, diplomacia de redes (supone participación flexible en organismos multilaterales para resolver problemas compartidos), diplomacia parlamentaria (en el marco de la cual fueron organizados dos Foros internacionales "Desarrollo del Parlamentarismo" en Moscú en 2018 y 2019).
} 
hasta la fecha de hoy, ascienden a cincuenta en total (Tratados internacionales de la Federación Rusa, 2019). No obstante, como en el caso de España, hay razones para creer que no todos los convenios están en la web. Del mismo modo, aparecen convenios sobre la protección de la propiedad intelectual en el desarrollo de las actividades de cooperación técnica militar, pero no existe -o es difícil de encontrar- alguna constancia de los propios tratados de cooperación técnica militar. Estos, con toda la lógica, deben ser previos a los de la protección de la propiedad intelectual en el dicho ámbito. También hay que destacar que en caso de Rusia algunos tratados de cooperación técnico-militar o militar, p.e. con China e India, fueron hallados en la web oficial del Ministerio de Asuntos Exteriores (pero no aparecen en el Portal oficial de actas del derecho internacional). Los convenios de cooperación en materia de defensa, en mayor parte, se centran en los países asiáticos, árabes y africanos. Pocos han sido firmados con América Latina y en Europa son solo cinco: Bielorrusia, Eslovenia, Grecia, Italia y Serbia. Lo que resulta notorio, a mi juicio, es la existencia de un convenio con Corea del Norte, sancionada económicamente y políticamente por todos los países del mundo.

No se puede decir mucho al respecto de las misiones de paz o cursos de formación militar, especialmente si comparamos con el nivel de desarrollo al que llegaron en España. En Rusia no existen como tales, pero hay algunas variedades nacionales que resultan interesantes para referirse. Las políticas y las actividades referentes a la enseñanza militar pueden agruparse en cuatro conjuntos: tratados para la docencia en las escuelas militares para niños extranjeros, maniobras militares, juegos militares y las colaboraciones del Ministerio para la Protección Civil, Situaciones de Emergencia y Eliminación de las Consecuencias de los Desastres Naturales (MchS en ruso).

La enseñanza a los niños extranjeros en las escuelas de primaria y secundaria que dependen del Ministerio de defensa es un campo relativamente nuevo para Rusia ,y los tratados consiguientes fueron firmados en los últimos cinco años con Siria, Osetia del Sur y Tayikistán.

Las maniobras militares rusas no difieren mucho, en su consistencia, de las que practican otros países. Pueden ser bilaterales o multilaterales, y siempre tienen el objetivo de verificar la calidad de repuesta a una situación de crisis inesperada. Hasta los acontecimientos ocurridos en Ucrania (2014), las maniobras se efectuaban con Noruega, Italia, Grecia, Francia, países bálticos o incluso con los EE.UU. En el período comprendido entre 2015 y 2019, las maniobras bilaterales se llevaron con China, India, Bielorrusia, Pakistán y Egipto, y las multilaterales con la Organización del Tratado de Seguridad Colectiva (OTSC). En el marco de las maniobras pueden ejercerse dos tipos de actividades aparte: lecciones y competiciones. Al mismo tiempo, se estrenan las noveades rusas en productos de uso militar (Lista de maniobras, 2019).

Los juegos militares, iniciados en 2014, no tienen análogos en el mundo. Empezaron con el campeonato en biatlón de tanques y con el tiempo se diversificaron hasta 32 tipos de torneos, entre los cuales destacan las luchas navales, aéreas, las competiciones de tropas de desembarco, de francotiradores, de artillería, de equipos para tratar amenazas químicas y bacteriológicas, de médicos, e incluso, de cocineros. En 2019 en los juegos participaron cuarenta países, la mayoría de los cuales fueron de Asia, África y Oriente Medio. Dos países fueron de América Latina: Cuba y Venezuela; y dos de Europa: Grecia y Serbia (Juegos militares, 2019). La mayoría de los torneos se celebra en el territorio ruso, y el propósito de 
invitar a los extranjeros a participar en una de las formas del pasatiempo predilectas en Rusia, que es competir en un torneo, no es el propósito central. Todo el equipamiento y las municiones durante los juegos son los productos de la industria militar rusa. Los militares extranjeros pueden probar en práctica el funcionamiento de estos "artilugios", lo que puede conducir a la firma de contratos de su exportación.

El Ministerio para la Protección Civil, Situaciones de Emergencia y Eliminación de las Consecuencias de los Desastres Naturales, conocido en Rusia por el acrónimo corto MChS, es el análogo a la Unidad de Emergencias española. Las principales vías de cooperación en el extranjero se centran en las ayudas para superar situaciones de crisis, participación en misiones humanitarias, de búsqueda y rescate, promoción de la metodología del Grupo internacional consultorio sobre los temas de búsqueda y rescate y coordinación de acciones en situaciones de crisis en el marco de la Organización de Naciones Unidas (ONU) (Cooperación internacional, 2020). La institución tiene su propia academia donde imparte programas de grado, posgrado y cursos cortos, y también está abierta a los extranjeros (Relaciones internacionales, 2020).

La cooperación en los temas de las tecnologías militares para Rusia representa uno de los ámbitos más profundizados. Si hablamos del branding del país en el mundo, en la mente surgen asociaciones socio-culturales (el ballet y el arte) e industriales (cosmoáutica/astronáutica y el Complejo de Industria Militar, CIM). La demanda a la seguridad por los gobiernos de muchos países la convierte en uno de los productos que se puede vender (por la vía de municiones y equipamiento, o por la vía del "avalo", al promover la imagen del país fuerte, pero abierto a la cooperación, y amistoso). Como sostiene Danilova (2017: 102-103):

La transformación y modernización militar rusa, la consolidación del poder armamentístico, los avances tecnológicos... despiertan el interés (en el mundo)... Se analiza la subida presupuestaria para el Programa estatal de armamento, la situación con el desarrollo activo de las Fuerzas armadas terrestres, aéreas, navales, sistemas de defensa antimisil (S-400 "Triumf", "Iscander") que está condicionada por la política del Presidente Vladimir Putin en las regiones del Sur y el Norte Occidental.

Los países que tradicionalmente compran a Rusia los productos militares pertenecen a las regiones de Oriente y de Asia. No obstante, los problemas y tendencias que condicionan las ventas del CIM son universales:

1. Crece el volumen de las ventas de productos militares a escala global;

2. Se demanda estos productos de más calidad;

3. Existe el riesgo de que más tarde pueden ser mejorados o $\operatorname{copiados}^{10}$;

4. Los países compradores buscan maneras de adquirir licencias para la producción. Como consecuencia, crean condiciones favorables para la inversión extranjera en este sector y desarrollan actividades de espionaje industrial;

\footnotetext{
${ }^{10}$ Es por eso que Rusia tardó muchos años en firmar un contrato sobre los suministros de materiales militares con China.
} 
5. En los contratos de cooperación técnica-militar a los proveedores se les demanda la inversión a las industrias militares nacionales ${ }^{11}$ (Gubin, 2016).

\section{A modo de conclusión.}

La teoría económica entre las funciones principales del Estado destaca las políticas de coerción, de redistribución y de defensa. El mantenimiento de la seguridad dentro de las fronteras nacionales, como el mantenimiento de estabilidad del sistema internacional, es el trofeo por el que siempre han luchado y siguen luchando los gobiernos nacionales. Hoy en día tenemos el mundo "empequeñecido" por la Globalización y el progreso tecnológico, que está proclamando los valores universales y está disputando por su desarrollo y aplicación. La solidez de la "marca nacional", es decir, el atractivo de la imagen del Estado, la fuerza del servicio diplomático, tiene mucho que ver con los poderes que goza el Estado en la arena internacional, cuestionando la práctica rudimentaria de contar solo con los arsenales nucleares. Este fenómeno de dispersión del poder y de alteración de roles tradicionales de los agentes tuvo lugar en todos los aspectos a los que estamos acostumbrados. La mayoría de los países occidentales han apostado a la cooperación internacional en el ámbito de defensa a través de la firma de los tratados correspondientes, entrenamiento y adiestramiento de las fuerzas policiales y militares de otros países sin importar los bloques de seguridad colectiva, ayudas a resolver problemas de seguridad regionales e incorporación a la nueva política exterior del Ministerio de Defensa.

En caso de Rusia observamos, al contrario, el enfoque arcaico, cuyo desarrollo máximo está representado por el sector del Complejo de Industria Militar (CIM). Las iniciativas de entrenamiento conjunto con otros países, las competiciones militares, la cooperación internacional del Ministerio de Emergencias Ruso carecen de una visión global y coordinada que permita desarrollar una revisada y reforzada "marca rusa". La necesidad de tener esa imagen nueva resulta crítica en estos momentos, dados los choques y la hostilidad extendiéndose por las diferencias entre las narraciones rusas y occidentales globales, y por las fakes news. La experiencia española, en este sentido, puede resultar interesante para el análisis ya que en su momento este país Ibérico tuvo que afrontar los mismos problemas de la defunción de la imagen negativa en el mundo provocada por la "leyenda negra"12. Para realizar la tarea fueron incorporados muchos agentes en la política exterior: desde las FAS para la resolución post conflicto en Centroamérica en los años 1990, hasta el Instituto Cervantes y el Instituto Elcano que trabajó a principios de los años 2000 en las fórmulas de Spain is different y Spain: the friend in Europe (Yakovlev, 2011:37). Tal vez este es el camino que tiene que considerar el gobierno ruso cuando este año (2020) va a revisar los conceptos clave de la política exterior y de la seguridad.

\footnotetext{
${ }^{11}$ Por ejemplo, las autoridades indias durante las negociaciones con Rusia de la compra-venta de aviones insistieron en que el $50 \%$ de todos los trabajos y servicios relacionados fueran realizados por las empresas indias.

12 El diccionario de la Real Academia Española define el término como "Relato desfavorable y generalmente infundado sobre alguien o algo" (DLE, 2020). Relacionamos la aparición de este concepto con el nombre de Juan Juderías que publicó el libro "La leyenda negra" en 1914, en el que hablaba de "la imagen denigrante de España" relacionada con la colonización de América Latina en la época de los Habsburgo españoles en el siglo XVI.
} 


\section{Bibliografía.}

Azuara Blanco, I. (2009), Diplomacia de Defensa, Boletín de Información, Ministerios de Defensa, №. 308, pp.73-116, available at: https://dialnet.unirioja.es/servlet/articulo?codigo=3090622 accessed on: 5 Enero 2020;

Bartolomé, M.C. La Diplomacia de Defensa y el caso de la Unidad Militar de Emergencias (UME) de España, Instituto de Relaciones Internacionales available at: http://www.iri.edu.ar/images/Documentos/boletinsegydef/boletin6/art_bartolome.pdf accessed on: 5 Enero 2020;

Cooperación internacional (2020), Ministerio para la Protección Civíl, Situaciones de Emergencia y Eliminación de las Consecuencias de los Desastres Naturales available at: https://www.mchs.gov.ru/deyatelnost/mezhdunarodnoe-sotrudnichestvo accessed on: 22 Enero 2020;

Cosialls Ubach, A.M. (2016) Diplomacia de Defensa: concepto y modelos, Diplomacia de Defensa, La Defensa en la Acción Exterior del Estado, Documento de trabajo 01/2016, IEEE. available at: http://www.ieee.es/Galerias/fichero/docs trabajo/2016/DIEEET01-2016 DiplomaciaDefensa.pdf accessed on: 22 Enero 2020;

Danilova, E.A. (2017) Comparative analysis of the international armed forces branding and Russian national branding of innovations in the defense industry as a response to the global challenges of our time (en ruso), Vlast', №7, pp.92-107;

Diccionario de la lengua española (2020), Leyenda available at: https://dle.rae.es/leyenda accessed on: 23 Enero 2020;

Doctrina Militar de la Federación Rusa (2014), Decreto del Presidente de la Federación Rusa, available at: http://pravo.gov.ru/proxy/ips/?docbody=\&prevDoc=102065541\&backlink=1\&\&nd=102135800 accessed on: 22 Enero 2020;

Estrategia de acción exterior (2015) Ministerio de Asuntos Exteriores y de Cooperación available at: http://www.exteriores.gob.es/Portal/es/SalaDePrensa/Multimedia/Publicaciones/Documents/ESTRATEGIA\%20DE\%20ACCION\%20EXTERIOR\%20castellano.pdf accessed on: 22 Enero 2020;

Exportaciones españolas de material de defensa, de otro material y de productos y tecnologías de doble uso en el primer semestre de 2018 (2019), Ministerio de Industria, Comercio y Turismo de España available at: http://www.comercio.gob.es/es-ES/comercio-exterior/informacion-sectorial/material-de-defensa-y-de-doble-uso/PDF/publicaciones/XMDDU2018I.pdf accessed on: 10 Enero 2020;

Fukuyama, F. (1989), The End of History? The National Interest, Summer 1989, pp. 1-18, available at: https://www.embl.de/aboutus/science society/discussion/discussion 2006/ref1-22june06.pdf accessed on: 10 Diciembre 2019;

Gubin, A.V. (2016), Military aspects of russia's involvement into East Asian political processes (en ruso), Oikumena, №4, pp.62-74;

Guía de tratados bilaterales con Estados (2019), Ministerio de Asuntos Exteriores y Cooperación, 04/07/2019 Available at: 
http://www.exteriores.gob.es/Portal/es/SalaDePrensa/Multimedia/Publicaciones/Documents/GUIA\%20TRATADOS\%20CON\%20PAISES.PDF accessed on: 22 Diciembre 2019;

Juegos militares (2019), available at: http://armygames2019.mil.ru/\#/ accessed on: 8 Noviembre 2019;

Lista de maniobras (2019), Ministerio de Defensa de la Federación de Rusia available at: https://structure.mil.ru/mission/practice/all.htm accessed on: 8 Noviembre 2019;

Ministerio de Industria, Comercio y Turismo, Gobierno de España available at: http://www.comercio.gob.es/es-ES/comercio-exterior/informacion-sectorial/material-de-defensa-y-de-doble-uso/PDF/publicaciones/XMDDU2018I.pdf accessed on: 8 Noviembre 2019;

Misiones en el exterior (2020), Ministerio de Defensa del Reino de España available at: https://www.defensa.gob.es/misiones/en exterior/ accessed on: 10 Enero 2020;

Moliner González, J.A. (2016), El concepto español de diplomacia de defensa, Diplomacia de Defensa, La Defensa en la Acción Exterior del Estado, IEEE, Documento de trabajo 01/2016, pp.73-94 available at: http://www.ieee.es/Galerias/fichero/docs trabajo/2016/DIEEET01-2016 DiplomaciaDefensa.pdf accessed on: 15 Octubre 2019;

Nossel, S. (2004), Smart Power, Foreign Affairs, March/April 2004 available at: https://www.foreignaffairs.com/articles/united-states/2004-03-01/smartpower accessed on: 10 Diciembre 2019;

Parte a Irak el contingente de la Guardia Civil (2017), Ministerio de Defensa Español available at: https://www.defensa.com/espana/parte-irak-contingente-guardia-civil accessed on: 10 Diciembre 2019;

Plan de Diplomacia de Defensa, Ministerio de Defensa del Reino de España available at: https://www.defensa.gob.es/Galerias/misiones/diplomaciaDefensadoc/DGL110506-Plan-Diplomacia.pdf accessed on: 15 Octubre 2019;

Podberezkin, A. (2016) Military force and the policy of the new public diplomacy, Observer, 12/2016, pp.15-25;

Referencia del Consejo de Ministros (2018) available at: https://www.lamoncloa.gob.es/consejodeministros/referencias/Paginas/2018/refc20181228.aspx accessed on: 15 Octubre 2019;

Relaciones internacionales (2020), Academia de la Protección Civil del MChs available at: https://amchs.ru/about the university/mezhdunarodnaya-deyatelnost/accessed on: 22 Enero 2020;

Sánchez Herráez, P. (2016) La presencia militar en la red político-dimplomática, Diplomacia de Defensa, La Defensa en la Acción Exterior del Estado, Documento de trabajo 01/2016, IEEE, 2016, pp. 95-122 available at: http://www.ieee.es/Galerias/fichero/docs trabajo/2016/DIEEET01-2016 DiplomaciaDefensa.pdf accessed on: 15 Octubre 2019; 
Sanz Roldán, F. (2000), La diplomacia de defensa: una aproximación desde España, Arbor CLXV, 651 (Marzo 2000), pp.519-527;

Sistema estatal de información de derecho available at: http://publication.pravo.gov.ru/SignatoryAuthority/international accessed on: 15 Octubre 2019;

The Strategic Defense Review White Paper (1998), Parliament of the United Kingdom, available at: https://researchbriefings.parliament.uk/ResearchBriefing/Summary/RP98-91 accessed on: 10 Diciembre 2019;

Tratados internacionales de la Federación Rusa (2019), Web-portal de información oficial, Sistema estatal de información de derecho available at: http://publication.pravo.gov.ru/SignatoryAuthority/international accessed on: 15 Octubre 2019;

Tuzhikov, Ye. y Pavlov, A. (2016) About development of the draft of the concept of defensive diplomacy of the Russian Federation (en ruso), Strateguicheskaya stabil'nost', №3(76), pp.69-75;

Yakovlev, P.P. (2011) España en la política mundial (en ruso), Moscú: ILA RAN, 384p. 
Anexo 1. Acuerdos internacionales de España en el ámbito de cooperación en materias de Seguridad y Defensa

\begin{tabular}{|c|c|c|c|c|c|c|}
\hline 1 & 2 & 3 & 4 & 5 & 6 & 7 \\
\hline No & País & $\begin{array}{l}\text { Acuerdo sobre } \\
\text { cooperación en } \\
\text { materia de } \\
\text { defensa }\end{array}$ & $\begin{array}{c}\text { Protocolo de } \\
\text { asistencia técnica en } \\
\text { materia de defensa y } \\
\text { seguridad }\end{array}$ & $\begin{array}{c}\text { Acuerdo de cooperación } \\
\text { para materiales de defensa } \\
\text { o intercambio de datos con } \\
\text { esos fines }\end{array}$ & $\begin{array}{l}\text { Protección de } \\
\text { información clasificada } \\
\text { relacionada con la } \\
\text { industria de defensa }\end{array}$ & $\begin{array}{c}\text { Protección de } \\
\text { información clasificada }\end{array}$ \\
\hline \multicolumn{7}{|c|}{ EUROPA } \\
\hline 3 & Austria & & & & & 2011 (2013) \\
\hline \multirow[t]{2}{*}{4} & \multirow[t]{2}{*}{ Bélgica } & 1985 (1987); & & & & \\
\hline & & 1988 (1990)* & & & & \\
\hline 5 & $\begin{array}{l}\text { Bosnia y } \\
\text { Herzegovina }\end{array}$ & $2006 \bullet$ & & & & 2017 (2018) \\
\hline 8 & Eslovaquia & 1999• & & & & 2009 (2010) \\
\hline 9 & Eslovenia & $1997 \bullet$ & & & 2014 (2015) & \\
\hline 10 & Estonia & $1999 \bullet$ & & & & 2005 (2007) \\
\hline 11 & Finlandia & & & & & 2009 (2010) \\
\hline \multirow[t]{2}{*}{12} & \multirow[t]{2}{*}{ Francia } & 1983 (1985); & & & & \multirow[t]{2}{*}{2006 (2007) } \\
\hline & & $1988(1990)^{*}$ & & & & \\
\hline 13 & Grecia & 1985 (1987) & & & 1990 (1992) & \\
\hline 14 & Hungría & 1995• & & & & 2016 (2017) \\
\hline
\end{tabular}




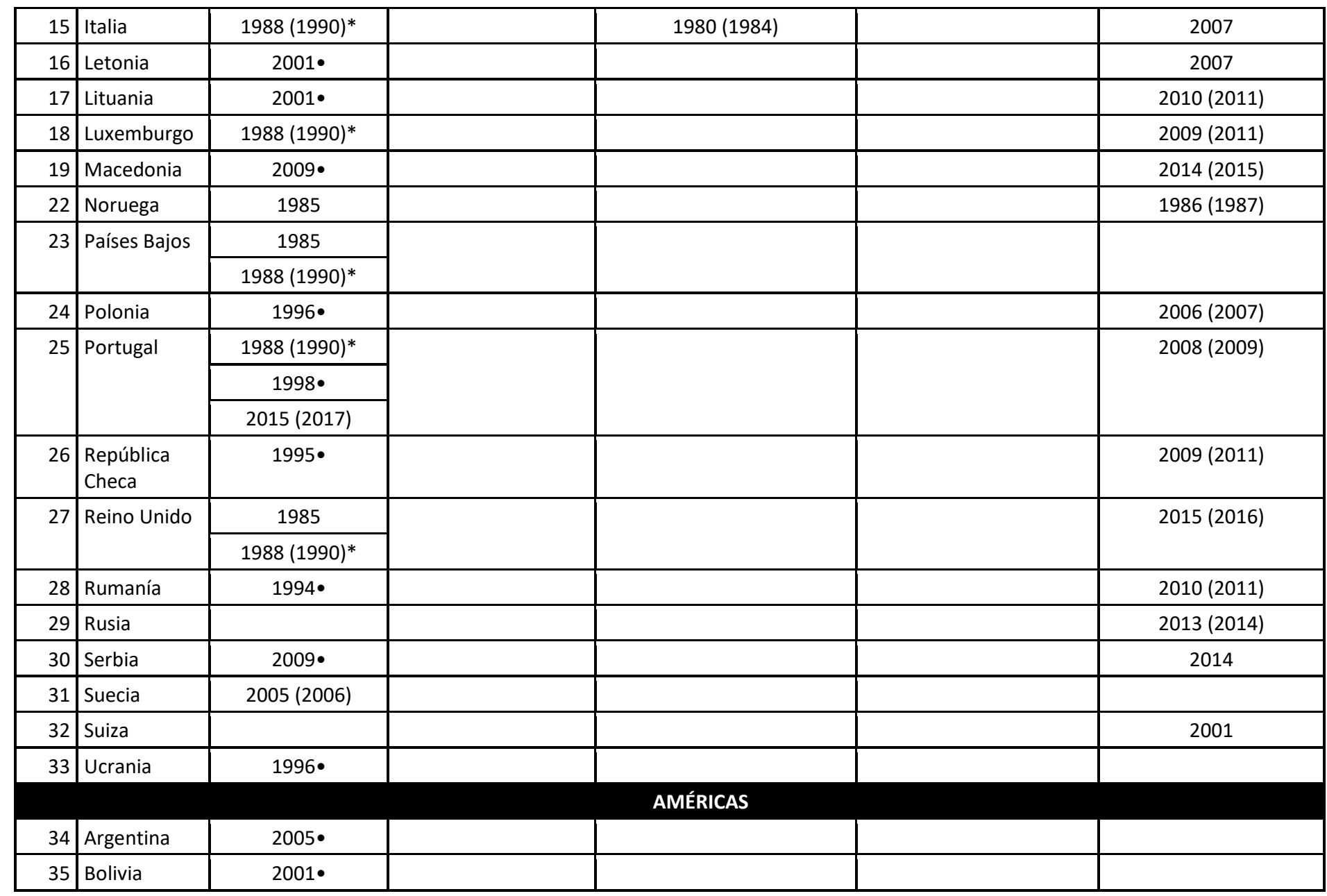




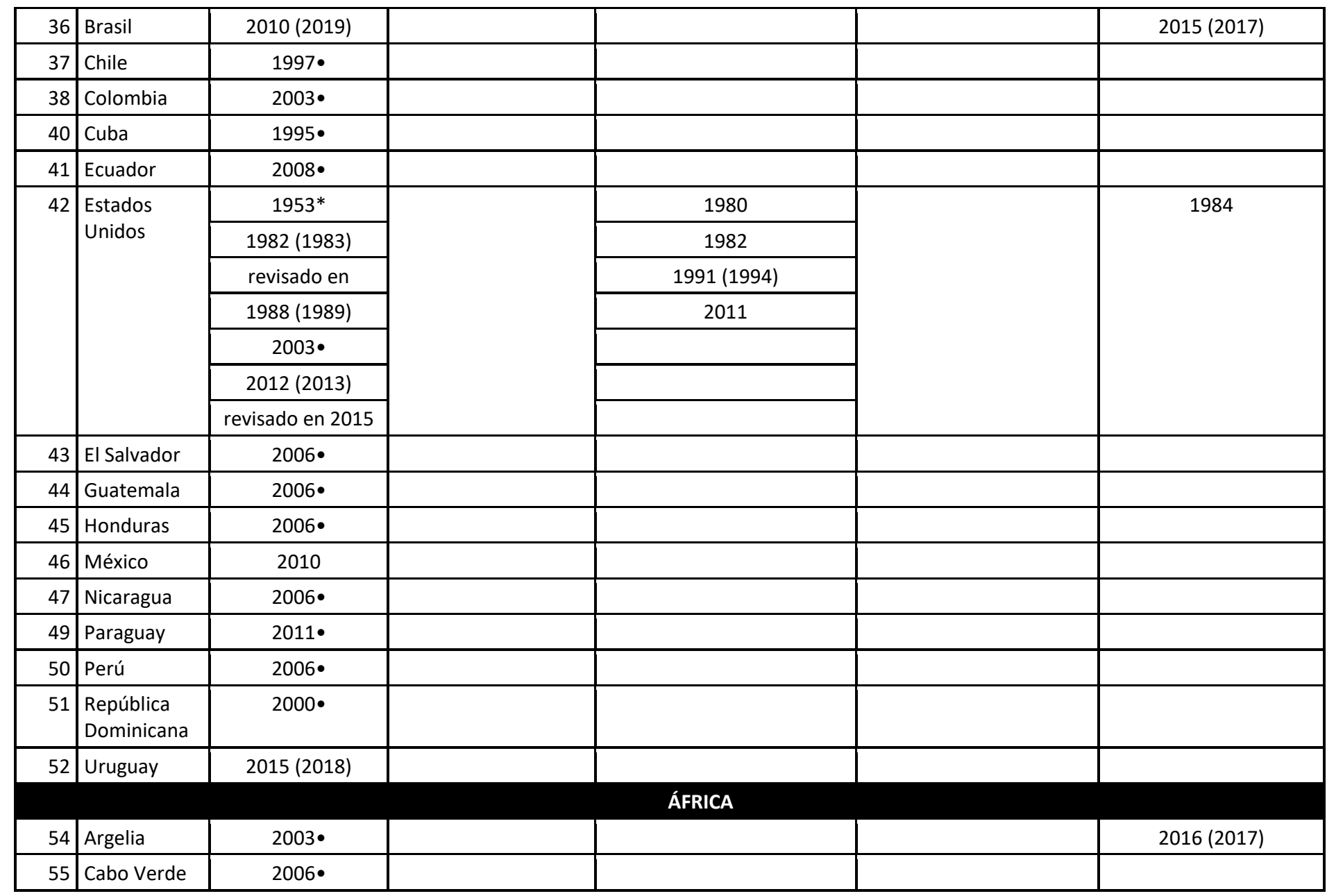




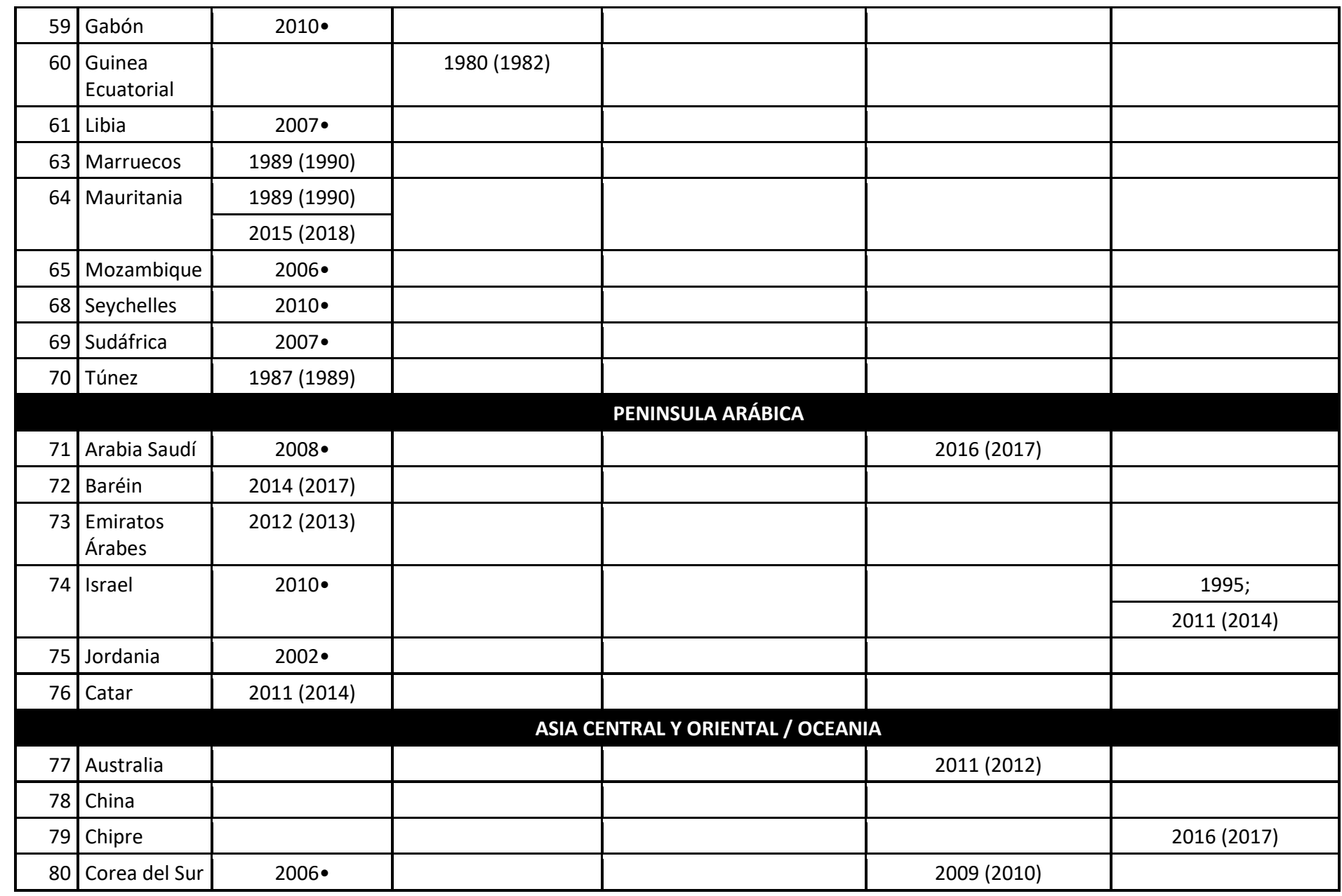




\begin{tabular}{|r|l|l|l|l|l|l|}
\hline 81 & Georgia & & & & & 2013 (2014) \\
\hline 82 & India & & & & & $2015(2016)$ \\
\hline 83 & Kazajistán & & & & & $2017(2018)$ \\
\hline 84 & Malasia & $2004 \bullet$ & & & & \\
\hline 85 & Singapur & & & & $2014(2015)$ & \\
\hline 86 & Turquía & & & & & $2014(2018)$ \\
\hline 87 & Vietnam & $2010 \bullet$ & & & & \\
\hline
\end{tabular}

Fuente: elaboración propia a partir de la Guía de tratados bilaterales con Estados. Ministerio de Asuntos Exteriores y Cooperación. 04/07/2019. Available at:

http://www.exteriores.gob.es/Portal/es/SalaDePrensa/Multimedia/Publicaciones/Documents/GUIA\%20TRATADOS\%20CON\%20PAISES.PDF (accessed 20.12.2019), y Plan de Diplomacia de Defensa. Ministerio de Defensa, 2011. Available at: https://www.defensa.gob.es/Galerias/misiones/diplomaciaDefensadoc/DGL-110506-PlanDiplomacia.pdf (accessed 20.12.2019).

Notas:

* Acuerdos de legítima defensa colectiva (mutua defensa con los EE UU).

- Estos acuerdos figuran en el Plan de Diplomacia de Defensa (2011) pero están auscentes en la Guía de tratados bilaterales. Tampoco se ha podido encontrar alguna constancia de ellos en el Boletín Oficial del Estado (www.boe.es).

() Entre los paréntesis redondos figura el año de la entrada en vigor. 
Anexo 2. Acuerdos internacionales de Rusia en el ámbito de cooperación en materias de Seguridad y Defensa

\begin{tabular}{|c|c|c|c|c|c|c|c|}
\hline 1 & 2 & & 3 & 4 & 5 & 6 & 7 \\
\hline No & País & $\begin{array}{l}\text { Cooperación } \\
\text { militar }\end{array}$ & $\begin{array}{c}\text { Cooperación técnico- } \\
\text { militar }\end{array}$ & $\begin{array}{l}\text { Protección de la } \\
\text { propiedad } \\
\text { intelectual (sobre } \\
\text { la protección } \\
\text { mutua de los } \\
\text { resultados de las } \\
\text { actividades } \\
\text { intelectuales y } \\
\text { protección la } \\
\text { propiedad } \\
\text { intelectual en el } \\
\text { marco de } \\
\text { cooperación } \\
\text { técnico-militar } \\
\text { bilateral) }\end{array}$ & $\begin{array}{l}\text { Convenios en } \\
\text { protección de la } \\
\text { información } \\
\text { secreta }\end{array}$ & $\begin{array}{l}\text { Control por la } \\
\text { existencia y el } \\
\text { uso específico de } \\
\text { los productos de } \\
\text { uso militar }\end{array}$ & Otro \\
\hline 2 & Eslovenia & & & & & $\begin{array}{c}2013(2014)+ \\
\text { intercambio de la } \\
\text { información } \\
\text { operativa }\end{array}$ & \\
\hline 3 & Grecia & & & & & & $\begin{array}{c}2013 \text { (2016): sobre la } \\
\text { compra de los } \\
\text { productos de uso } \\
\text { militar }\end{array}$ \\
\hline 4 & Italia & & & 2007 (2009) & & & \\
\hline
\end{tabular}




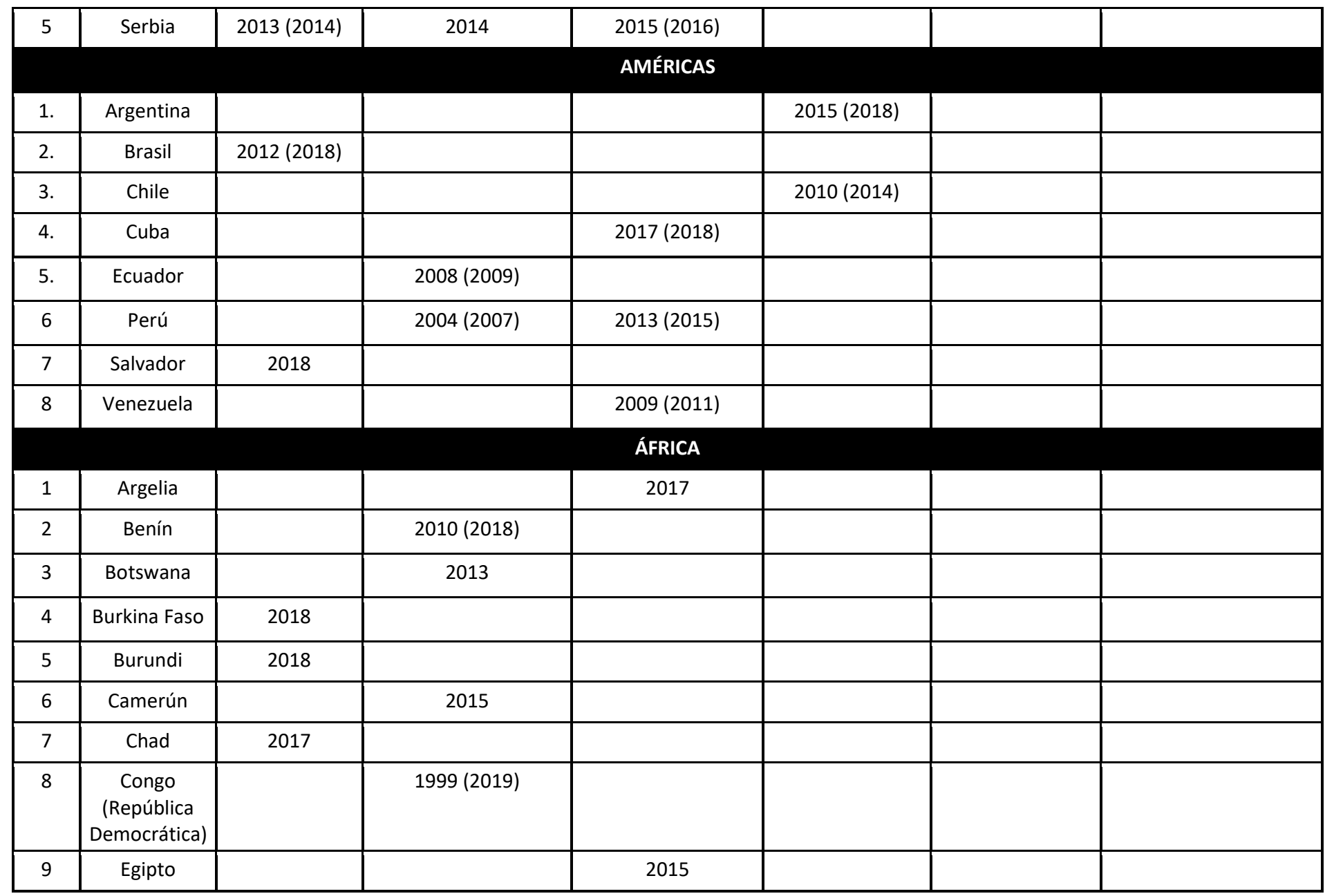

236 Relaciones Internacionales - № 58/2020 


\begin{tabular}{|c|c|c|c|c|c|c|c|}
\hline 10. & Madagascar & 2018 & & & & & \\
\hline 11. & Malí & 2019 & & & & & \\
\hline 12. & Marruecos & & & 2017 & 2016 & & \\
\hline 13. & Mozambique & & 2015 (2016) & & & & \\
\hline 14. & Níger & 2017 & 2017 (2018) & & & & \\
\hline 15. & Nigeria & 2017 & & & & & \\
\hline 16. & Ruanda & & 2016 (2017) & 2019 & & & \\
\hline 17. & Suazilandia & 2017 & 2017 & & & & \\
\hline 18. & Sudán & 2018 & & & & & \\
\hline 19. & Tanzania & & 2018 & & & & \\
\hline 20. & Túnez & & 2016 (2017) & & & & \\
\hline 21. & Uganda & & 2003 (2019) & & & & \\
\hline 22. & Yibuti & & 2012 (2013) & & & & \\
\hline 23. & Zimbabue & & 1999, 2015 & 2019 & & & \\
\hline \multicolumn{8}{|c|}{ PENÍNSULA ARÁBICA } \\
\hline 1 & Baréin & 2016 & & & & & \\
\hline 2 & Catar & & 2017 & & & & \\
\hline 3 & $\begin{array}{c}\text { Emiratos } \\
\text { Árabes } \\
\text { Unidos }\end{array}$ & & & 2015 (2016) & & & \\
\hline 4 & Líbano & 2018 & 2010 (2012) & & & & \\
\hline
\end{tabular}




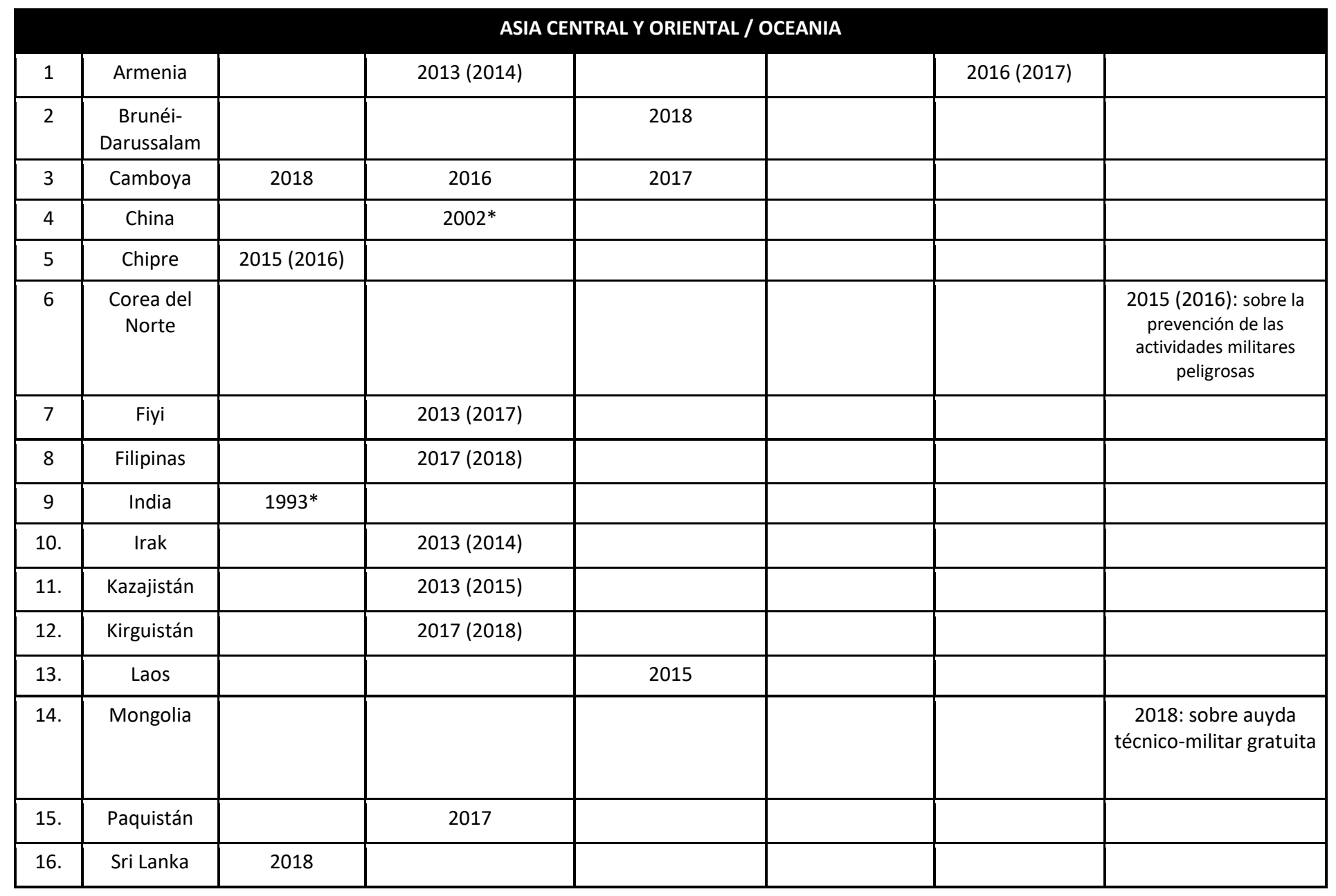




\begin{tabular}{|c|c|c|c|c|c|c|c|}
\hline 17. & Tailandia & 2016 & 2017 & & & \\
\hline 18. & Tayikistán & & $1994(2001)$ & & & & \\
\hline 19. & Uzbekistán & & $2016(2017)$ & & & $2016(2018)$ & \\
\hline 20. & Vietnam & 2013 & & $2010(2014)$ & & & \\
\hline
\end{tabular}

Fuente: elaboración propia a partir del Web-portal de información oficial, Sistema estatal de información de derecho available at:

http://publication.pravo.gov.ru/SignatoryAuthority/international accessed on: 15.10.2019 Notas: * Acuerdos que figuran en la web del Ministerio de Asuntos

Exterioires de la Federación de Rusia (https://www.mid.ru/foreign_policy/international_contracts/2_contract) () Entre los paréntesis redondos figura el año de la entrada en vigor. 
Consistently ranked at the top of its field, International Security has defined the debate on security policy for more than forty years

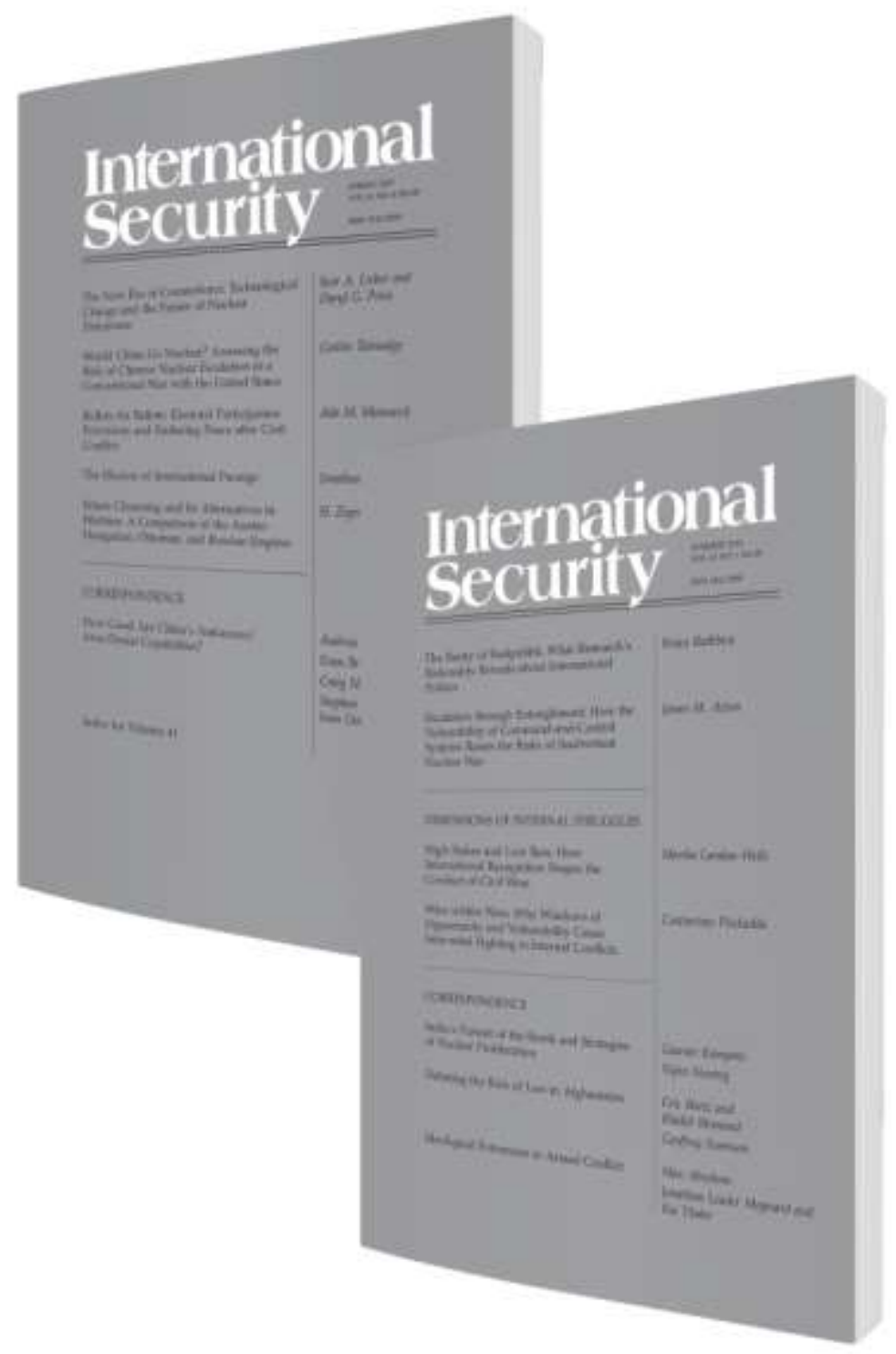

mitpressjournals.org/isec 